\title{
Adaptive Shadow Detection Using a Blackbody Radiator Model
}

\author{
Aliaksei Makarau, Rudolf Richter, Rupert Müller, and Peter Reinartz
}

\begin{abstract}
The application potential of remotely sensed optical imagery is boosted through the increase in spatial resolution, and new analysis, interpretation, classification, and change detection methods are developed. Together with all the advantages, shadows are more present in such images, particularly in urban areas. This may lead to errors during data processing. The task of automatic shadow detection is still a current research topic. Since image acquisition is influenced by many factors such as sensor type, sun elevation and acquisition time, geographical coordinates of the scene, conditions and contents of the atmosphere, etc., the acquired imagery has highly varying intensity and spectral characteristics. The variance of these characteristics often leads to errors, using standard shadow detection methods. Moreover, for some scenes, these methods are inapplicable. In this paper, we present an alternative robust method for shadow detection. The method is based on the physical properties of a blackbody radiator. Instead of static methods, this method adaptively calculates the parameters for a particular scene and allows one to work with many different sensors and images obtained with different illumination conditions. Experimental assessment illustrates significant improvement for shadow detection on typical multispectral sensors in comparison to other shadow detection methods. Examples, as well as quantitative assessment of the results, are presented for Landsat-7 Enhanced Thematic Mapper Plus, IKONOS, WorldView-2, and the German Aerospace Center (DLR) 3K Camera airborne system.
\end{abstract}

Index Terms-Blackbody radiator, multispectral image, Planck equation, shadow detection.

\section{INTRODUCTION}

$\mathbf{S}$ HADOWING is one of the main and inevitable acquisition artifacts in high-resolution optical data. The quality of data processing may be significantly degraded by the appearance of shadows, particularly in urban areas. Since images are obtained in different areas of the Earth and with different conditions of the atmosphere, the intensity and the spectral characteristics of the imagery have high variations, and the task of an automatic shadow detection becomes very complex. Prevention of errors caused by this kind of artifacts is still a current topic and discussed widely in remote sensing literature.

Manuscript received August 23, 2010; revised October 27, 2010; accepted November 16, 2010. Date of publication January 12, 2011; date of current version May 20, 2011. This work was supported by the German Aerospace Center and German Academic Exchange Service (DLR-DAAD) postdoctoral fellowship under Award A/09/95629.

The authors are with the German Aerospace Center (DLR), 82234 Wessling, Germany (e-mail: aliaksei.makarau@dlr.de; rudolf.richter@dlr.de; rupert.mueller@dlr.de; Peter.Reinartz@dlr.de).

Digital Object Identifier 10.1109/TGRS.2010.2096515
The applied methods on shadow detection may be divided into several broad groups of methods based on the following: 1) physical properties of light propagation/geometry [1]; 2) color-invariant models (color space transformation) [2]-[4]; and 3) computational and statistical models [5]-[9].

Since the methods based on the physics of light propagation and the sun-object-sensor geometry are designed for specific applications, they are difficult to apply to real scenes [10]. Therefore, the methods based on the properties of shadowed areas are preferred.

Tappen et al. [7] proposed a method that uses multiple cues to recover shading from a single image. A classifier is trained to recognize gray-scale patterns on an image derivative. Each image derivative is classified as being caused by shading or a change in the surface's reflectance. The classifier gathers local evidence about the surface form and color, which is then propagated using the generalized belief propagation algorithm.

In the paper of $\mathrm{Wu}$ and Tang [9], the shadow extraction problem is analyzed and formulated using a Bayesian framework. The method requires user's input, and the supplied hints are employed to effectively impose useful constraints for solving the difficult and ill-posed shadow extraction problem from one image.

The hierarchical algorithm proposed by Yao and Zhang [6] consists of two levels of processing: the pixel level classification (achieved through modeling an image as a reliable graph) and on maximization of the graph reliability using the expectation-maximization algorithm. The region verification is achieved through minimizing the Bayesian error by further exploiting the domain knowledge.

Tsai [2] assesses the transformation of red, green, and blue (RGB) color image into different invariant color spaces to decouple chroma and luma components. Otsu method is used to segment shadow. Chung et al. [3] presented a modification of this method with local shadow thresholding. Transformation into different color spaces can modify color invariance properties; therefore, such approaches can fail on complex scenes.

Salvador et al. [4] proposed another method of cast shadow detection for still and moving images. This method exploits the spectral and geometrical properties of the shadowing process. A hypothesis is applied on the fact that cast shadows darken the surface which they are cast upon, and color invariance with geometric properties of shadows is used to verify detected regions. The information integration stage confirms or rejects the initial hypothesis.

Polidorio et al. [11] proposed a robust technique by thresholding the difference image of the saturation and the intensity component in a normalized hue, saturation, and intensity color 
space. Considering the atmospheric Rayleigh scattering effect, this technique allows one to segment shadowed areas in color images obtained by airborne and orbital sensors.

Tian et al. proposed a tricolor attenuation model for shadow detection [10]. The parameters of the model are fixed by using the spectral power distributions of daylight and skylight, which are precalculated and fixed. Based on the model, a multistep shadow detection algorithm is proposed to extract shadows. The authors report that the weakness of this method is that it will fail on detecting shadows in sunrise and sunset (the correlated color temperatures during sunrise and sunset are very different from the correlated color temperatures used in the paper [10]). Since this method has static parameters, it can fail on remotely sensed data obtained at different daytimes with varying latitude/longitude (Lat/Lon) coordinates and atmospheric conditions.

In this paper, we propose an alternative method for shadow detection using a blackbody radiator model. This approach is fully motivated by the physical process of shadow formation. Since all the parameters of the method are calculated directly from the input data, this method is adaptive and performs on all remotely sensed optical data (medium-resolution, highresolution, and very high resolution airborne and spaceborne sensors). The numerical assessment using ground truth data with several types of shadow situations illustrates the high performance of the approach.

This paper is organized as follows: In Section II, the physical model of shadow formation is presented, together with an application of the blackbody radiator model for shadow detection. An automatic parameter set calculation by solution of the equations is derived in Section III. A step-by-step algorithm is presented in Section IV. Experiments on different data, numerical evaluation using ground truth, and comparison with other methods are shown in Section V, and the assessment of the results and discussion are presented in Section VI.

\section{Model of Shadow Formation}

Illumination of an outdoor area is characterized by two main light components: direct sunlight and the atmospheric scattered light (diffuse skylight). The diffusion of sunlight in the atmosphere is caused by Rayleigh and aerosol scatterings. Obscuring objects from the direct sunlight causes the appearance of shadows, and the objects in this area are illuminated by the scattered light. We take the common assumption that the nonshadowed parts of the scene are illuminated by direct sunlight, but shadowed regions are illuminated only by the scattered light [12] (Fig. 1).

Objects of the scene can have varying reflection characteristics and can be illuminated by different types of illumination (direct sunlight and/or scattered light). Therefore, besides the different reflection of objects, the recorded intensities in the image may have a very high variation. Instead of the recorded image intensities to be used for shadow detection, the properties of the direct sunlight and the scattered-light illumination sources are expected to provide a more stable and robust way for shadow detection. Calculation of the illumination source properties instead of using image intensities allows the separa-

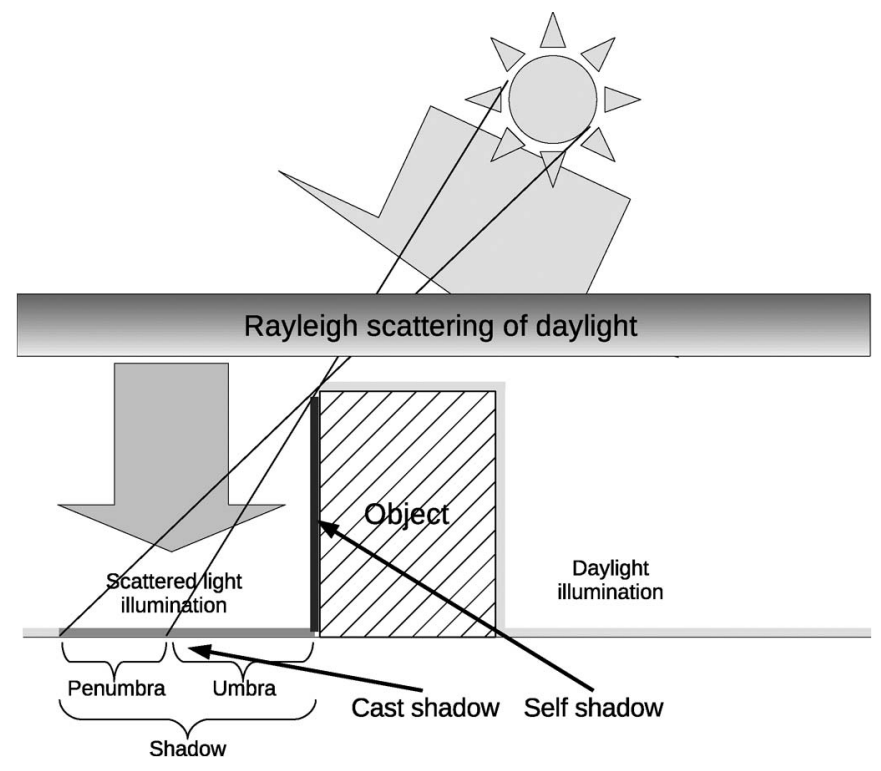

Fig. 1. Shadowed area formation. The area under sunlight illumination is characterized by daylight sun illumination spectra, while the shadowed area is characterized primarily by the spectra of the scattered sunlight.

tion of two areas in the scene: the area illuminated by direct sunlight and the shadowed area (i.e., illuminated by scattered light).

\section{A. Image Acquisition}

We assume a Lambertian surface reflection model for the image-forming process. The intensity values recorded by a digital camera (three spectral channels) can be described as

$$
p_{k}=\tau \int_{\omega}\left[T(\lambda) S(\lambda) E(\lambda) / \pi+L_{p}(\lambda)\right] Q_{k}(\lambda) d \lambda
$$

where $p_{k}$ is the recorded intensity (digital number) in a color channel $k=R, G, B$ (RGB channel); $\tau$ is the gain factor that is dependent on the camera aperture, electronics, and the integration time; $T(\lambda)$ is the total ground-to-sensor transmittance; $S(\lambda)$ is the surface spectral reflectance; $E(\lambda)$ is the spectral irradiance at ground level; $Q_{k}(\lambda)$ is the sensitivity of the color sensor; and $L_{p}(\lambda)$ is the atmospheric path radiance.

For atmospherically corrected data, $p_{k}$ is already evaluated at the ground

$$
p_{k}^{\mathrm{atm}}=\tau \int_{\omega}\left[S(\lambda) E(\lambda) Q_{k}(\lambda) / \pi\right] d \lambda .
$$

Then, several assumptions and approximations are taken. A narrow camera model is assumed (narrow bandwidths) with center wavelength $\lambda_{k}$ for each channel [13], [14]. Under the assumption of calibrated camera, $S$ becomes constant for each channel [13]. We can reasonably approximate (2) into

$$
I_{k}=\tau S_{k} E_{k},\left(S_{k}=S\left(\lambda_{k}\right), E_{k}=E\left(\lambda_{k}\right)\right)
$$

where $I_{k}$ is the intensity in the image (approximated $p_{k}^{\text {atm }}$ ) and $E_{k}$ includes the factor $\pi$ for brevity. The intensities in the acquired image can have a very high variation. To represent color in an invariant way (instead of color triplets), many researchers [13], [14] proposed to use color chromaticities. Color 
chromaticities are widely used in the literature on color science, color constancy, and shadow detection and compensation.

The formation of recorded color chromaticity $i_{k}$ is represented by multiplication of surface and illumination chromaticities $s_{k}$ and $e_{k}$

$$
i_{k}=s_{k} e_{k}, \quad(k=r, g) .
$$

Moreover, color chromaticities in an image may be obtained by the ratios of the $R, G$, or $B$ values to the sum of the $R, G$, and $B$ values [15] or by the ratios of the $R$ and $G$ values to the $B$ value [13]. We use chromaticities as a ratio of the $R$ and $G$ values to the $B$ value

$$
i_{r}=I_{R} / I_{B} \quad i_{g}=I_{G} / I_{B}
$$

The dependence of a material chromaticity on the recorded intensity can be shown by substituting (3) into (5) (note that $\tau$ is canceled out)

$$
\begin{gathered}
s_{r}=S_{R} / S_{B}=\frac{I_{R} E_{B}}{E_{R} I_{B}} \\
s_{g}=S_{G} / S_{B}=\frac{I_{G} E_{B}}{E_{G} I_{B}} .
\end{gathered}
$$

\section{B. Illuminant Approximation by the Blackbody Radiator Model}

The next assumption is that illumination spectra can be approximated by the model of a blackbody radiator [13]. Blackbody radiator model is found very useful for direct-sunlight spectra modeling in the applications on color constancy [13]-[15].

Planck's formula for a blackbody emitting spectral radiance is defined in the following way:

$$
M(\lambda, T)=c_{1} \lambda^{-5}\left[\exp \left(c_{2} / T \lambda\right)-1\right]^{-1}
$$

where $M(\lambda, T)$ is the spectral power of the blackbody radiation, $c_{1}$ and $c_{2}$ are constants $\left(c_{1}=3.7418 \times 10^{-16} \mathrm{~W} \cdot \mathrm{m}^{2}\right.$, and $\left.c_{2}=1.4388 \times 10^{-2} \mathrm{mK}\right), \lambda$ is the wavelength $m$, and $T$ is the temperature in Kelvin [14].

The blackbody radiator model can be used to represent the illuminant's chromaticity

$$
\begin{aligned}
& e_{r}(T)=\frac{M\left(\lambda_{R}, T\right)}{M\left(\lambda_{B}, T\right)} \\
& e_{g}(T)=\frac{M\left(\lambda_{G}, T\right)}{M\left(\lambda_{B}, T\right)}
\end{aligned}
$$

or using (7)

$$
\begin{array}{r}
e_{r}(T)=\frac{\lambda_{B}^{5}\left[\exp \left(\frac{c_{2}}{T \lambda_{B}}\right)-1\right]}{\lambda_{R}^{5}\left[\exp \left(\frac{c_{2}}{T \lambda_{R}}\right)-1\right]} \\
e_{g}(T)=\frac{\lambda_{B}^{5}\left[\exp \left(\frac{c_{2}}{T \lambda_{B}}\right)-1\right]}{\lambda_{G}^{5}\left[\exp \left(\frac{c_{2}}{T \lambda_{G}}\right)-1\right]} .
\end{array}
$$

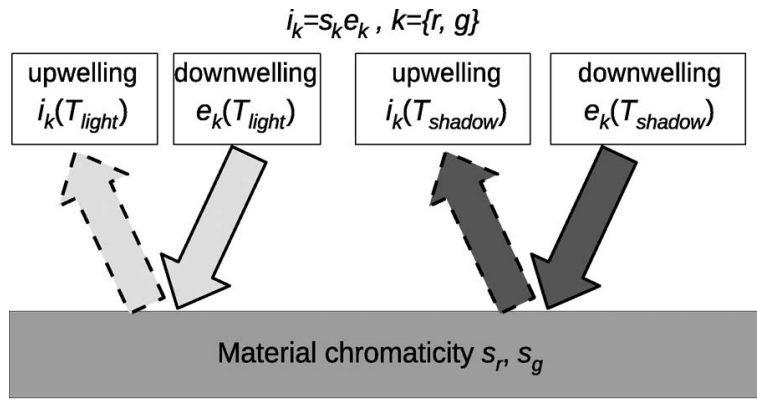

Fig. 2. Uniform material is illuminated by different sources of downwelling light: Direct sunlight and scattered light. The constant chromaticity of the material allows one to recover the chromaticities of the two illuminants and to calculate the temperatures of blackbody radiators.

The characteristics of direct sunlight and scattered skylight are dependent on the temperature of the blackbody radiator (the temperatures for direct sunlight and scattered skylight). Let us denote the temperature for direct sunlight as $T_{\text {light }}$ and the temperature for the scattered light (shadowed area) as $T_{\text {shadow }}$. It should be noted that $T_{\text {shadow }}>T_{\text {light }}$, since the spectra of scattered light are more bluish than the spectra of direct sunlight. Recovery of the temperatures allows one to identify the regions under sunlight illumination and scattered light illumination (shadowed region) independently of the scene.

\section{Shadow Detection Using the BLACKBODY RADIATOR MODEL}

To calculate the temperatures $T_{\text {shadow }}$ and $T_{\text {light }}$, we can use the fact that the same object in a scene can be under direct sunlight and can be shadowed. This means that the same material of the object is illuminated by the two different illuminants (Fig. 2). To find the temperatures, we can use the fact that the image chromaticities divided by the illuminant's chromaticity are identical to the surface chromaticity [14] (the surface chromaticity is the same under both direct-sunlight and scattered-light illuminants).

\section{A. Illuminant Temperature Calculation}

Using (4), (5), and (9), a set of equations can be established, and the temperatures of the blackbody illuminants can be calculated

$$
\frac{i_{r, \text { shadow }}}{e_{r, \text { shadow }}}-\frac{i_{r, \text { light }}}{e_{r, \text { light }}}=0
$$

where $i_{r, *}$ and $i_{g, *}$ are calculated using (5) or

$$
\frac{i_{g, \text { shadow }}}{e_{g, \text { shadow }}}-\frac{i_{g, \text { light }}}{e_{g, \text { light }}}=0
$$

where the indices "shadow" and "light" denote the chromaticities of the shadowed and illuminated areas of the material. The assumption for (10) and (11) is that the chromaticity of the 
material is constant. Components $e_{r, \text { shadow }}, e_{r, \text { light }}, e_{g \text {,shadow }}$, and $e_{g, \text { light }}$ are calculated by

$$
\begin{gathered}
e_{r, \text { shadow }}=\frac{\lambda_{B}^{5}}{\lambda_{R}^{5}} \frac{\left[\exp \left(\frac{c_{2}}{T_{\text {shadow }} \lambda_{B}}\right)-1\right]}{\left[\exp \left(\frac{c_{2}}{T_{\text {shadow }} \lambda_{R}}\right)-1\right]} \\
e_{r, \text { light }}=\frac{\lambda_{B}^{5}}{\lambda_{R}^{5}} \frac{\left[\exp \left(\frac{c_{2}}{T_{\text {light }} \lambda_{B}}\right)-1\right]}{\left[\exp \left(\frac{c_{2}}{T_{\text {light }} \lambda_{R}}\right)-1\right]} \\
e_{g, \text { shadow }}=\frac{\lambda_{B}^{5}}{\lambda_{G}^{5}} \frac{\left[\exp \left(\frac{c_{2}}{T_{\text {shadow }} \lambda_{B}}\right)-1\right]}{\left[\exp \left(\frac{c_{2}}{T_{\text {shadow }} \lambda_{G}}\right)-1\right]} \\
e_{g, \text { light }}=\frac{\lambda_{B}^{5}}{\lambda_{G}^{5}} \frac{\left[\exp \left(\frac{c_{2}}{T_{\text {light }} \lambda_{B}}\right)-1\right]}{\left[\exp \left(\frac{c_{2}}{T_{\text {light }} \lambda_{G}}\right)-1\right]} .
\end{gathered}
$$

Since the chromaticities of the direct-sunlight and scatteredlight illuminants are different, it is possible to perform a detection of shadow areas (the detection is dependent on the temperatures of blackbody radiators $T_{\text {light }}$ and $\left.T_{\text {shadow }}\right)$

$$
\left(i_{r} / e_{r, \text { shadow }}-i_{r} / e_{r, \text { light }}\right)<\text { thresh }{ }_{r}
$$

or

$$
\left(i_{g} / e_{g, \text { shadow }}-i_{g} / e_{g, \text { light }}\right)<\text { thresh }_{g}
$$

where thresh $_{r}$ and thresh $\mathrm{t}_{g}$ are the threshold parameters for shadow segmentation. Only one equation is necessary for the shadowed-region detection. The detection of the shadowed regions can be made using the $e_{r}$ chromaticity (12) or using $e_{g}$ chromaticity (13). The threshold value thresh (or thresh $_{g}$ ) can be calculated automatically by the Otsu method [16], from a functional dependence on two variables $T_{\text {light }}$ and $T_{\text {shadow }}$, or manually. The nonparametric and unsupervised Otsu method allows automatic threshold selection for image segmentation. An optimal threshold is calculated according to the shape of image histogram to separate the resultant classes in gray levels with the between-class variance maximization and the intraclass variance minimization. Manual parameter selection can be used in an offline method to reach the best possible quality of shadow detection.

\section{B. Equation Solution}

Since the functions of (10) and (11) describe an exponential dependence, the direct minimization of the squared sum is difficult to perform [14]. The authors in [14] propose to use bracketing for the solution. In this paper, numerical Brent's method was used (IDL function zbrent.pro with modifications) [17]. Brent's method combines root bracketing, bisection, and inverse quadratic interpolation to converge from the neighborhood of a zero crossing. Using this method, convergence is reached as long as the function is evaluated within the interval known to contain a root.

The initial values are set to constrain the ranges to the expected solution according to the prior knowledge of the temperature range for a particular illuminant. The approximate

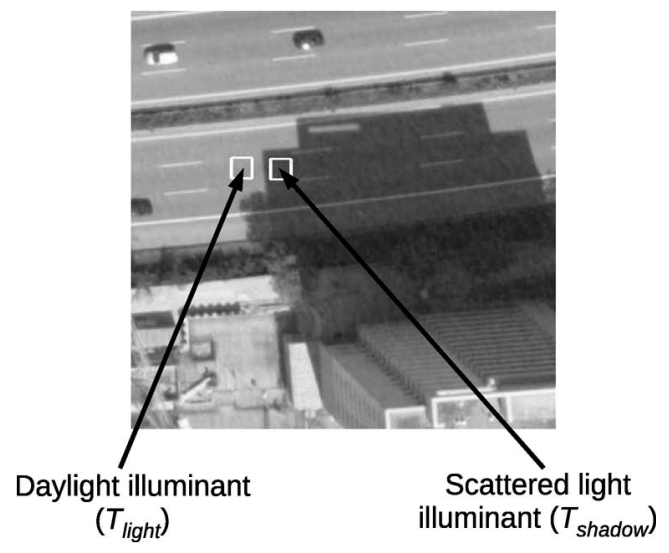

Fig. 3. Example of constant material (asphalt) partly shadowed and illuminated by direct sunlight. Regions under different illuminants (sunlight and scattered light) are outlined by rectangles.

temperature for the scattered light (blackbody radiator model) is in the range $7000-7500 \mathrm{~K}$, and that for direct sunlight is from 5500 to $6500 \mathrm{~K}$ [18]. For example, the temperature of sunrise/sunset illumination is in the range 2000-3000 K, while the temperature of clear blue sky is between 10000 and $20000 \mathrm{~K}$. The variation of the temperatures is possible due to varying Sun elevation, geographical coordinates, etc. We set extended ranges for the temperatures to include a wider range of possible values: $T_{\text {light }}$ is in the range $[5500,7000]$, and $T_{\text {shadow }}$ is in the range [7000, 8500].

To calculate image chromaticities $i_{r \text {,shadow }}$ and $i_{r, \text { light }}$ (or $i_{g \text {,shadow }}$ and $\left.i_{g \text {,light }}\right)$, the $R, G$, and $B$ pixel triplet values in two regions (illuminated and shadowed) of the same material have to be taken. An example of an object of uniform material (asphalt) illuminated by direct sunlight and partly shadowed is shown in Fig. 3.

The two regions are selected near a shadow border, at the shadowed and illuminated sides. The shadow border can be found in different ways (depending on the application of the method): Automatic approaches are described in [19]-[21] or manually. To make the calculation of the parameter set more robust, several pairs of pixel triplets are randomly selected from the two rectangular regions where the temperatures are calculated and the mean values of the $T_{\text {light }}$ and $T_{\text {shadow }}$ temperatures are used for further calculations.

\section{Shadow Detection Algorithm}

The step-by-step shadow detection algorithm is executed as follows.

1) Find the border of shadow irrespective of the location in the scene or shadow type. This can be done manually or automatically [19]-[21].

2) Locate shadowed and illuminated regions (see example in Fig. 3). To make sure that the shadowed and illuminated pixels are from the same object or material, pixels should be taken near the shadow border (in the shadow and illuminated parts).

3) Calculate chromaticities $i_{r \text {,shadow }}$ and $i_{r, \text { light }}$ (or $i_{g \text {,shadow }}$ and $\left.i_{g, \text { light }}\right)$ for the shadowed and illuminated regions using (5). 


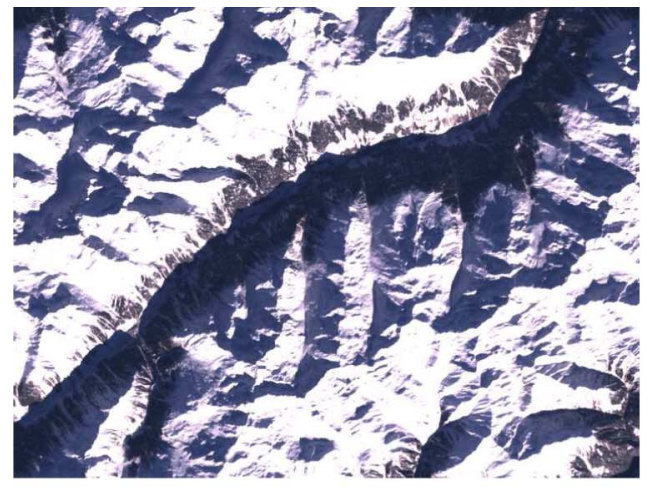

(a)

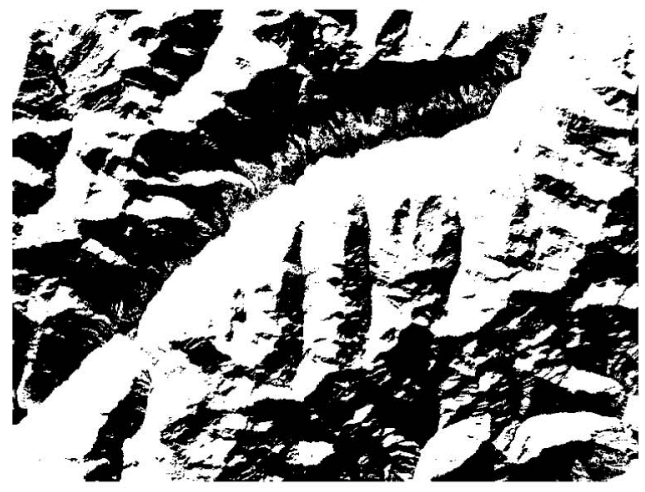

(c)

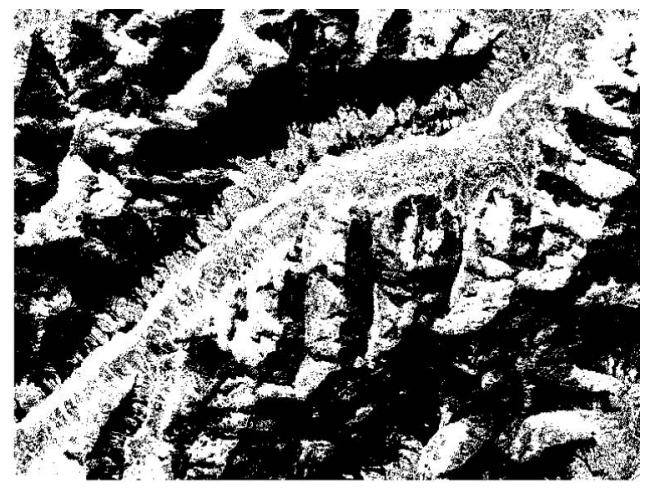

(b)

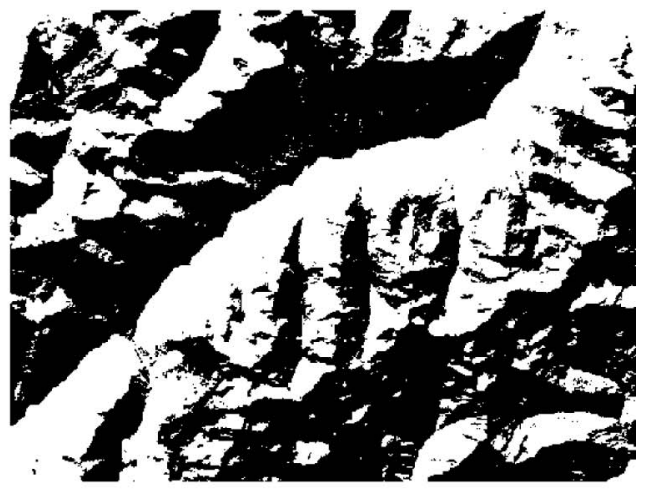

(d)

Fig. 4. Shadow detection on Landsat-7 ETM+ data (topographic shadows, mountain area, winter). (a) RGB band composition. (b) Tsai method [2]. (c) Shorter method [24]. (d) Proposed method. Shadow masks are represented in "white."

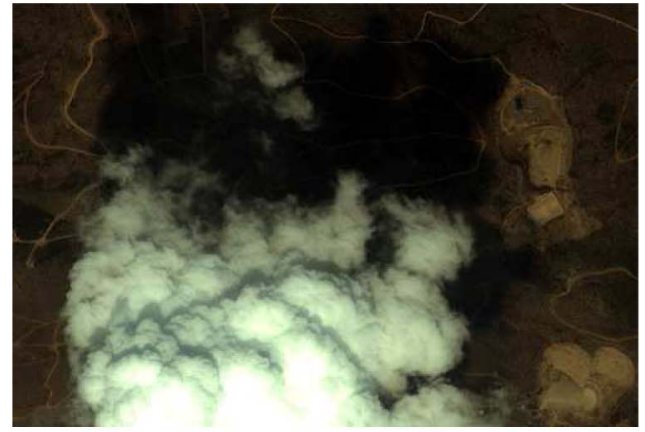

(a)

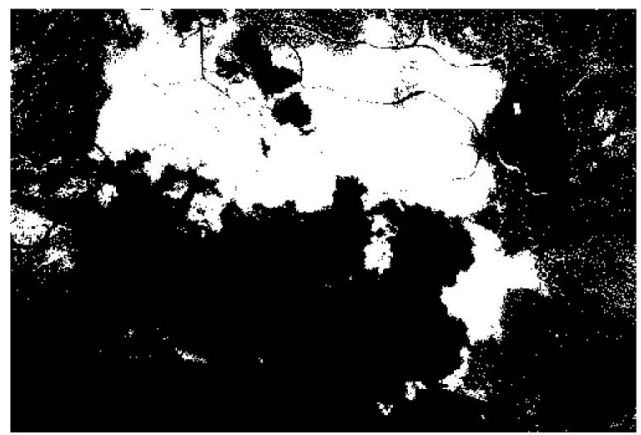

(c)

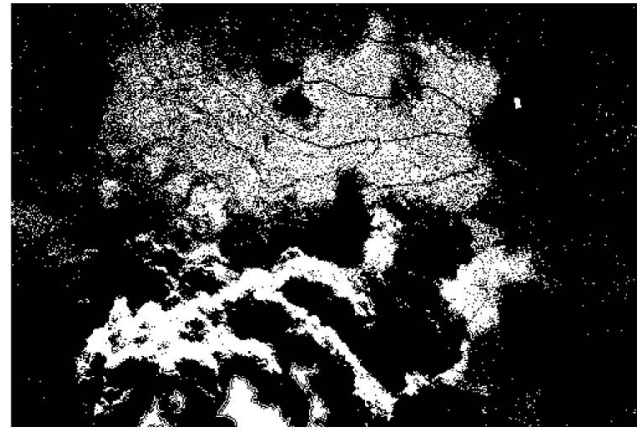

(b)

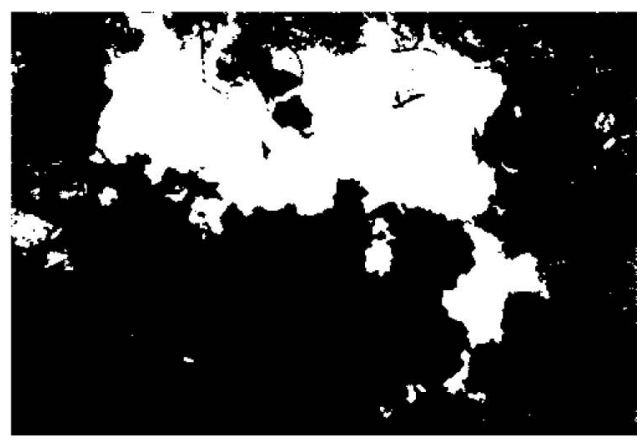

(d)

Fig. 5. Shadow detection on IKONOS data (cloud shadows, urban area, 4-m spatial resolution). (a) RGB band composition. (b) Tsai method [2]. (c) Shorter method [24]. (d) Proposed method. Shadow masks are represented in "white."

4) Calculate the parameters of the method $T_{\text {light }}$ and $T_{\text {shadow }}$ using (10) and (11) according to the temperature ranges for direct-sunlight and scattered-light illuminants.
5) Calculate the chromaticities $i_{r}$ (or $i_{q}$ ) for the image and the values of $e_{r \text {,shadow }}$ and $e_{r, \text { light }}$ (or $e_{g \text {,shadow }}$ and $\left.e_{g, \text { light }}\right)$ using (12) and (13), respectively. 
6) Calculate the mask for the shadowed regions using (14) or (15).

The adaptive part of the algorithm consists in the calculation of the temperatures $T_{\text {light }}$ and $T_{\text {shadow }}$ at step 4) (parameters of the method) for the particular multispectral image.

\section{Performance Evaluation}

This section presents a description of the used multispectral data, the metrics, and the results of numerical assessment and comparison of the developed method. Medium, high, and very high spatial resolution remotely sensed optical imageries were used for the numerical assessment of the proposed method.

Medium-resolution data sets included Landsat-7 Enhanced Thematic Mapper Plus (ETM+) scenes, high-resolution data sets comprised IKONOS scenes, and the very high resolution data sets were composed from the scenes acquired by WorldView-2 and the German Aerospace Center (DLR) 3K Camera system [22]. The spectral sensitivity ranges for the channels are presented in tables. The channels employed for shadow detection in the developed method are marked in italic.

In order to illustrate the robustness of the shadow detection method, different and commonly encountered shadowing situations and shadow types were used: 1) shadow caused by cloudiness (medium- and high-resolution data: Landsat-7 ETM+ and IKONOS); 2) shadow caused by buildings (very high resolution data: WorldView-2 and DLR 3K Camera system); and 3) topographic shadows (mountainous area and medium-resolution data: Landsat-7 ETM+). High-resolution and very high resolution data allow one to assess the method with increased accuracy.

To enable numerical evaluations of the developed method, test regions with different shadow types were cropped from the scenes. Manual interpretation of shadowed regions was made in order to create ground truth of shadow regions (true shadow masks). The true shadow masks were used in the evaluation of the precision of the shadow detection methods.

The performance of shadow detection methods is usually assessed by several metrics, among them the detection rate $(D R)$ and false-alarm rate $(F A R)$. These metrics are appropriate for an assessment of still shadow detection methods and depend on the true positives (TPs; the number of shadow points that are classified correctly), false negatives ( $F N$; the shadow points classified as background), and false positives ( $F P$; the background points are detected as shadows) [23]

$$
D R=\frac{T P}{T P+F N}
$$

(TP rate, or recall), and

$$
F A R=\frac{F P}{T P+F P}
$$

(corresponds to $1-p$, where $p$ is the so-called precision in the classification theory). The $D R$ is expected to increase up to a value of one, while the $F A R$ is expected to decrease to zero, respectively.
TABLE I

LANDSAT-7 ETM+ BAND SPECTRAL AND SPATIAL RESOLUTIONS

\begin{tabular}{|c|c|c|}
\hline Band (VNIR) & Spectral resolution, $\mu m$ & Spatial resolution, $m$ \\
\hline Band I (B) & $0.45-0.515$ & 30 \\
\hline Band 2 (G) & $0.525-0.605$ & 30 \\
\hline Band 3 (R) & $0.63-0.690$ & 30 \\
\hline Band 4 (NIR) & $0.75-0.90$ & 30 \\
\hline
\end{tabular}

In the following sections, we present several examples of widely used spaceborne and airborne imageries with common shadow situations, together with detection results. The scenes were acquired during different seasons and times, as well as in different geographical areas.

For comparison, two other recent and competitive methods were selected: the Victor Tsai method [2] and the Nicholas Shorter method [24]. Both methods show robust DRs for a broad range of applications. The threshold parameters in both methods were selected manually in such a way that the best possible quality of shadow detection can be reached.

The $i_{r}$ chromaticity and thresh $h_{r}$ threshold parameter were employed for shadow detection in the developed method. In comparison to the $i_{g}$ chromaticity, the $i_{r}$-chromaticity-based shadow detection allows one to use wider value range of the thresh $_{r}$ parameter and to calculate the thresh $h_{r}$ value more precisely.

The numerical assessment rates for all the detection methods are presented in Table IV.

\section{A. Landsat-7 ETM+}

This example contains topographic shadows in a mountain region [Switzerland, Fig. 4(a)]. The acquisition parameters are as follows: Lat/Lon: N46.43\%/E11.13 ${ }^{\circ}$, date: January 26, 2000, and time: UTC 09:45:00.

The threshold values are 135 and -0.35 for the Tsai and Shorter methods, respectively.

The calculated values for the proposed method parameter set are $T_{\text {shadow }}=8228 \mathrm{~K}, T_{\text {light }}=5519 \mathrm{~K}\left(\lambda_{B}=0.4787 \mu \mathrm{m}\right.$, $\lambda_{G}=0.561 \mu \mathrm{m}$, and $\left.\lambda_{R}=0.6614 \mu \mathrm{m}\right)$, and thresh th $=0.55$ (Table I)

\section{B. IKONOS}

For IKONOS imagery, we present results on cloud shadow detection [urban area, Athens, Greece, Figs. 5(a) and 6(a)]. The acquisition parameters are as follows: Lat/Lon: N38.04\% $/ \mathrm{E} 23.67^{\circ}$, date: July 27, 2004, and time: UTC 08:46:44.

The threshold values for the Tsai method are 45 [Fig. 5(b)] and 135 [Fig. 6(b)]. The threshold values for the Shorter method are -0.5 [Fig. 5(c)] and -0.37 [Fig. 6(c)].

The calculated values for the developed method parameter set are $T_{\text {shadow }}=7765 \mathrm{~K}, T_{\text {light }}=5984 \mathrm{~K}\left(\lambda_{B}=0.4805 \mu \mathrm{m}\right.$, $\lambda_{G}=0.5505 \mu \mathrm{m}$, and $\left.\lambda_{R}=0.665 \mu \mathrm{m}\right)$, and thresh th $=0.45$ (Table II).

\section{WorldView-2}

For WorldView-2 multispectral image, we present results on the detection of the shadows from buildings (urban area, 
TABLE II

IKONOS BAND SPECTRAL AND SPATIAL RESOLUTIONS

\begin{tabular}{|c|c|c|}
\hline Band (VNIR) & Spectral resolution, $\mu m$ & Spatial resolution, $m$ \\
\hline Band $1(B)$ & $0.445-0.516$ & 4 \\
\hline Band 2 (G) & $0.506-0.595$ & 4 \\
\hline Band 3 (R) & $0.632-0.698$ & 4 \\
\hline Band 4 (NIR) & $0.757-0.853$ & 4 \\
\hline
\end{tabular}

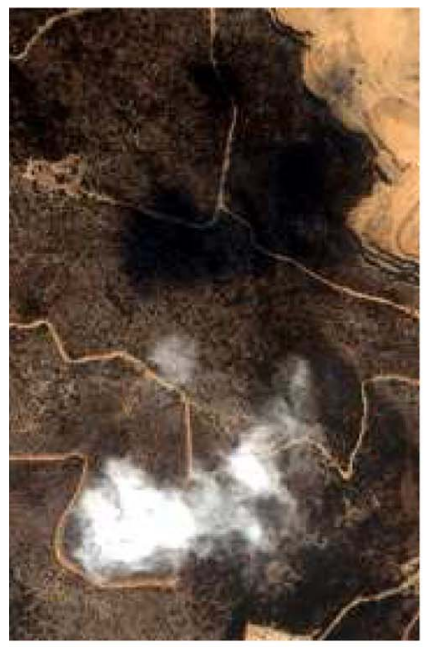

(a)

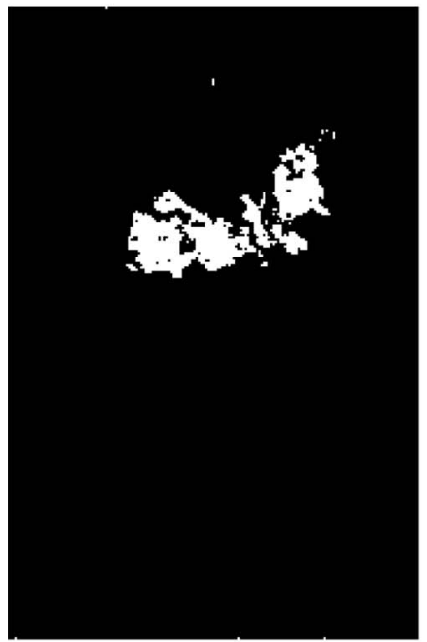

(c)

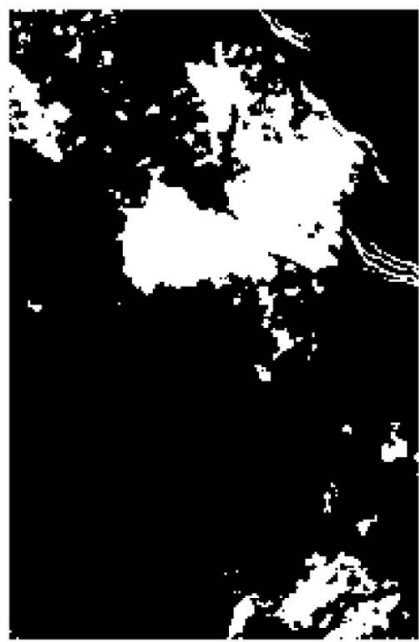

(d)

Fig. 6. Shadow detection on IKONOS data (cloud shadows, urban area, 4-m spatial resolution). (a) RGB band composition. (b) Tsai method [2]. (c) Shorter method [24]. (d) Proposed method. Shadow masks are represented in "white."

Munich, Germany, Fig. 7). The acquisition parameters are as follows: Lat/Lon: N48.13\%/E11.57 ${ }^{\circ}$ date: February 25, 2010, and time: UTC 10:22:06. The blue, green, and red bands were used for shadow detection.

The threshold values are 130 and -0.6 for the Tsai and Shorter methods, respectively.

The calculated values for the proposed method parameter set are $T_{\text {shadow }}=7962 \mathrm{~K}, T_{\text {light }}=6035 \mathrm{~K}\left(\lambda_{B}=0.4805 \mu \mathrm{m}\right.$, $\lambda_{G}=0.5505 \mu \mathrm{m}$, and $\left.\lambda_{R}=0.665 \mu \mathrm{m}\right)$, and thresh $h_{r}=0.5$ (Table III).

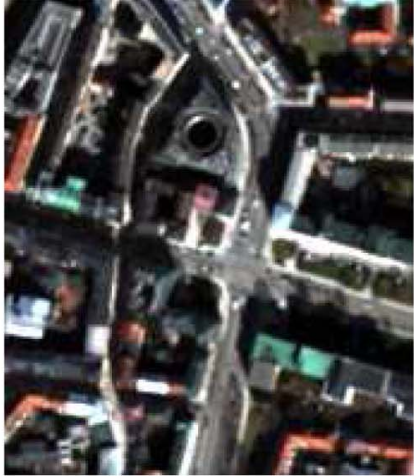

(a)

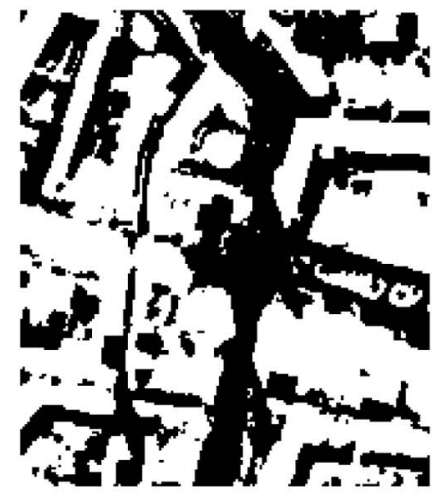

(c)

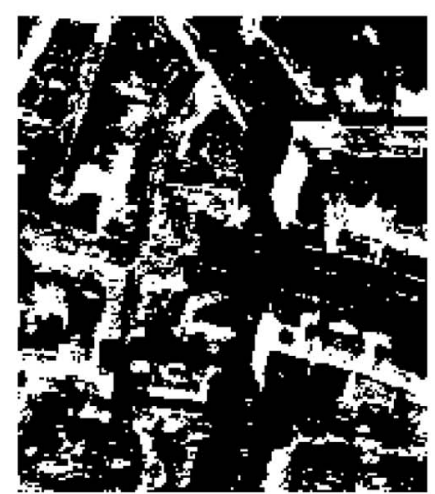

(b)

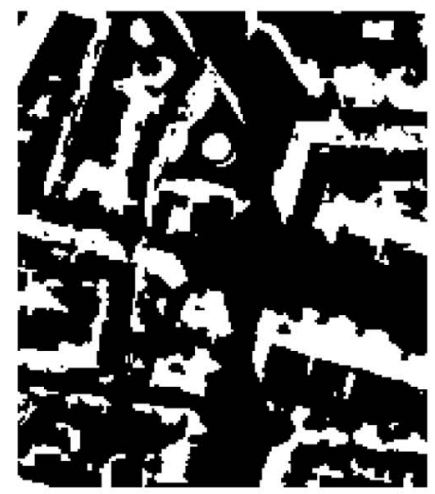

(d)
Fig. 7. Shadow detection on WorldView-2 data (urban area, 2-m spatial resolution). (a) RGB band composition. (b) Tsai method [2]. (c) Shorter method [24]. (d) Proposed method. Shadow masks are represented in "white."

TABLE III

WORLDVIEW-2 BAND SPECTRAL AND SPATIAL RESOlUtions

\begin{tabular}{|c|c|c|}
\hline Band & Spectral resolution, $\mu m$ & Spatial resolution, $m$ \\
\hline Band 1 (Coastal) & $0.400-0.450$ & 2 \\
\hline Band 2 (Blue) & $0.450-0.510$ & 2 \\
\hline Band 3 (Green) & $0.510-0.580$ & 2 \\
\hline Band 4 (Yellow) & $0.585-0.625$ & 2 \\
\hline Band 5 (Red) & $0.630-0.690$ & 2 \\
\hline Band 6 (Red edge) & $0.705-0.745$ & 2 \\
\hline Band 7 (NIR1) & $0.770-0.895$ & 2 \\
\hline Band 8 (NIR2) & $0.860-1.040$ & 2 \\
\hline
\end{tabular}

\section{DLR $3 K$ Camera}

For the DLR 3K Camera system, we evaluated the detection of shadows from buildings. Two types of test scenes were selected: shadows from low-rise buildings (residential area, scene 1) and shadows from high-rise buildings (scene 2).

For scene 1 (low-rise buildings, residential area) Munich, Germany [Fig. 8(a)], the acquisition parameters of the DLR 3K Camera airplane system are as follows: Lat/Lon: $\mathrm{N} 48.12^{\circ} / \mathrm{E} 11.48^{\circ}$, date: 30 April 2007, time: UTC 12:52:28, aperture: F9.11, exposure time: $1 / 2048$ seconds, altitude: $2466 \mathrm{~m}$, spatial resolution: $0.292 \mathrm{~m}$.

In Fig. 8, the threshold values are 135 and -0.37 for the Tsai and Shorter methods, respectively. In Fig. 9, the threshold values are 135 and -0.35 for the Tsai and Shorter methods, respectively. 


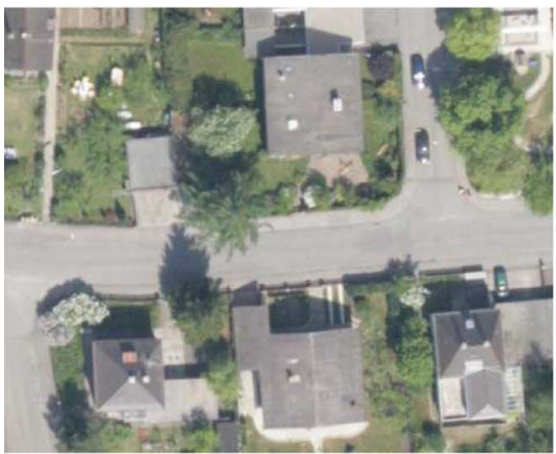

(a)

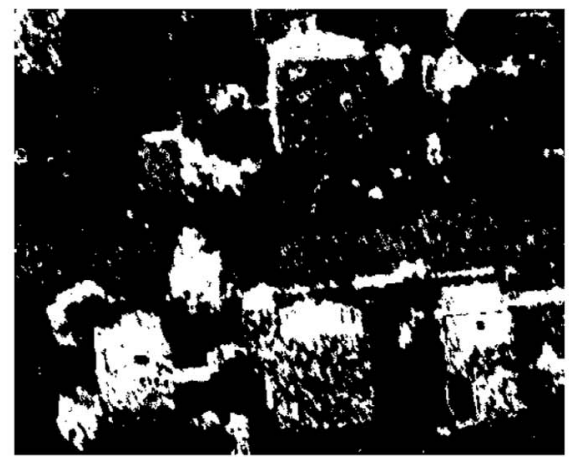

(b)

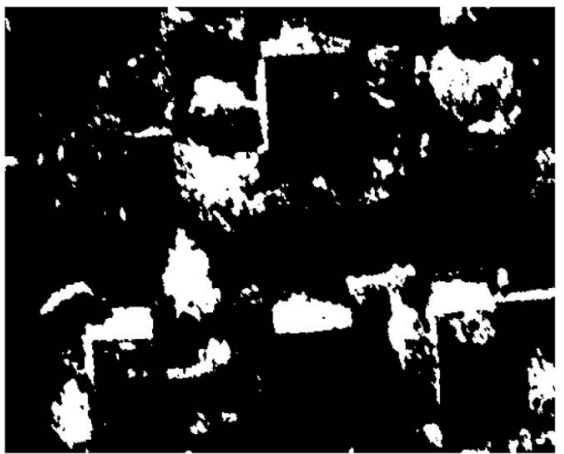

(c)

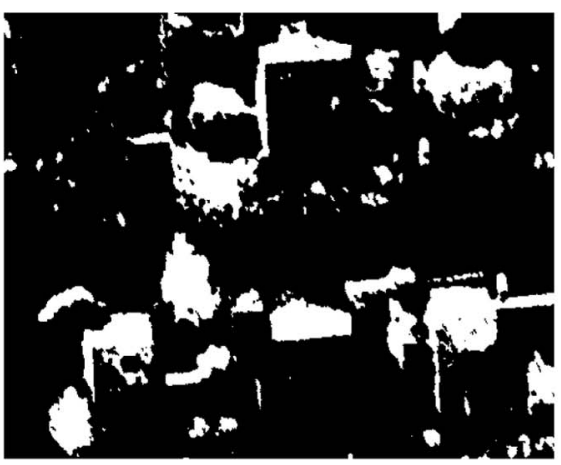

(d)

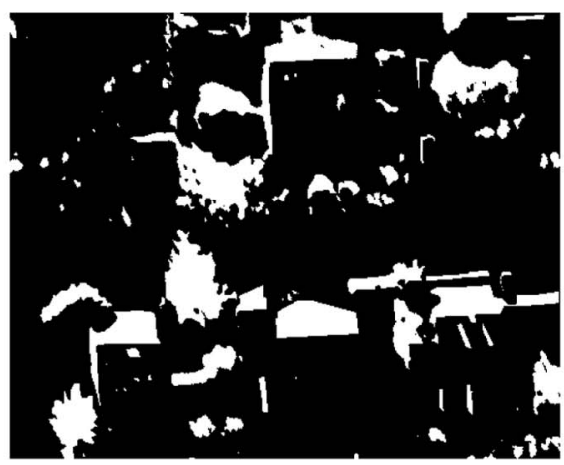

(e)

Fig. 8. Shadow detection on DLR 3K Camera data (shadows from low-rise buildings, residential urban area). (a) RGB band composition. (b) Tsai method [2]. (c) Shorter method [24]. (d) Proposed method. (e) Manually interpreted shadow mask. Shadow masks are represented in "white."

The calculated values for the proposed method parameter set are $T_{\text {shadow }}=8260 \mathrm{~K}, T_{\text {light }}=5993 \mathrm{~K},\left(\lambda_{B}=0.4787 \mu \mathrm{m}\right.$, $\lambda_{G}=0.561 \mu \mathrm{m}$, and $\lambda_{R}=0.6614 \mu \mathrm{m}$ were taken as for Landsat-7 ETM+; see Section VI), and thresh $h_{r}=0.55$.

For scene 2 (high-rise buildings, residential area), Munich, Germany [Fig. 9(a)], the acquisition parameters and the temperatures are the same as those for scene 1 .

\section{Results Assessment And Discussion}

The numerical evaluation of the method for scenes with complex objects provides high precision for shadowed-region detection (see Table IV). Examples of IKONOS test images (Figs. 5 and 6) contain roads in shadowed region. Despite the much higher intensity of the pixels of the roads, the shadowed area was detected with the best quality, which is one of the examples where other color transformation and segmentation methods may fail (e.g., Tsai method).

Another example on cast shadow detection is shown in Fig. 6. In this experiment, the illuminated and shadowed regions were taken at the border of the shadow from a cloud, and the values for the method parameter set were calculated. As a result, all cast shadows were detected, and the self-shadow (an object is shadowed by itself) of the mountain was also detected [see Fig. 6(d)]. This practical example illustrates that the shadowed regions can be detected irrespective of the shadow type (i.e., all types of shadows are detected in the scene), and the detection depends on the calculated temperatures of direct sunlight and the scattered-light sources. The Tsai and Shorter methods did not provide acceptable results and did not detect self-shadows.

Fig. 7 shows an RGB image composed from the WorldView2 spectral channels (visible range), together with the corresponding shadow masks. In this experiment, the illuminated region of a roof and the shadowed part (i.e., self-shadow) at the same roof were taken for the temperature calculations. Cast shadows and self-shadows were detected in the scene [Fig. 7(d)]. Shorter's method results in an increased value of the $F A R$ score, caused by the wrong interpretation of buildings as shadows.

For the DLR 3K Camera system, the central wavelengths were taken as for Landsat-7 ETM $+\left(\lambda_{B}=0.4787 \mu \mathrm{m}, \lambda_{G}=\right.$ $0.561 \mu \mathrm{m}$, and $\left.\lambda_{R}=0.6614 \mu \mathrm{m}\right)$. It was found that a small change of the central wavelengths does not degrade the accuracy of detection. All the methods provided little degradation of the DR and FAR (caused by moving cars, Figs. 8 and 9).

The DLR 3K Camera test sites illustrate the main drawbacks of the Tsai and Shorter methods. The Tsai method detects roofs of buildings as shadowed regions and is unable to deal with bright objects under shadow (Fig. 8, road surface marking). Shorter's method mistakes vegetation (trees in Figs. 8 and 9) for shadows and is unable to properly detect shadow over bright objects (Fig. 8, concrete pavement).

Table IV illustrates the advantage of the proposed method for all the examples having the highest DR and the lowest false acceptance rate FAR. In Fig. 7(a), the Shorter method provided 


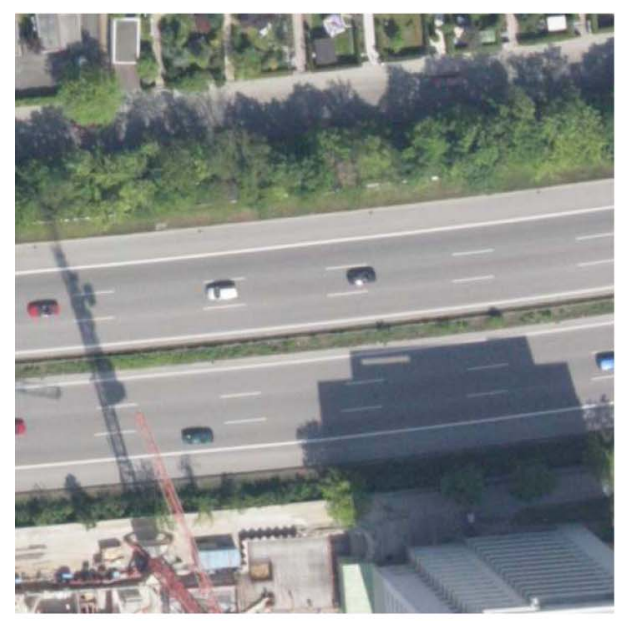

(a)

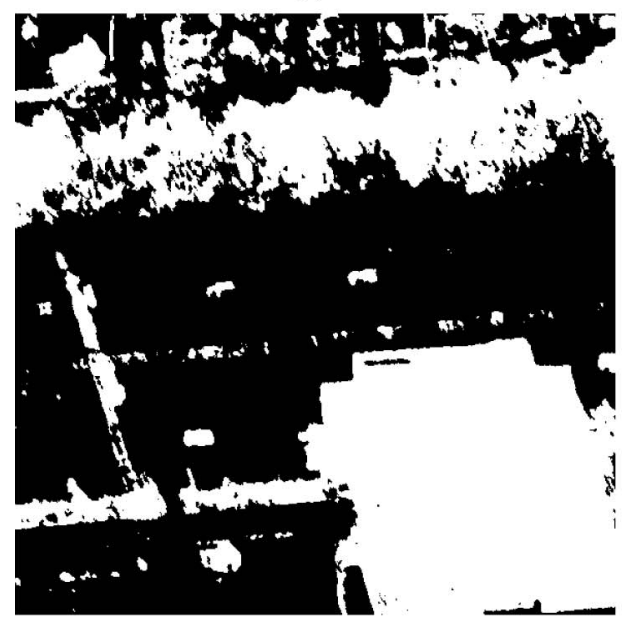

(c)

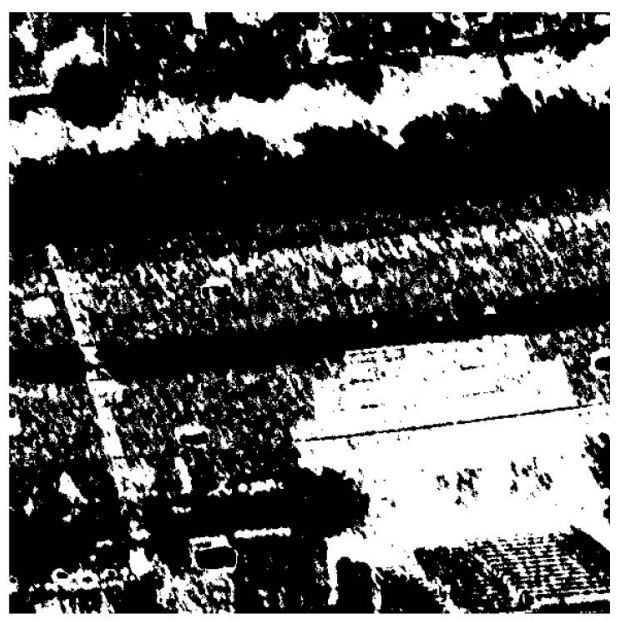

(b)

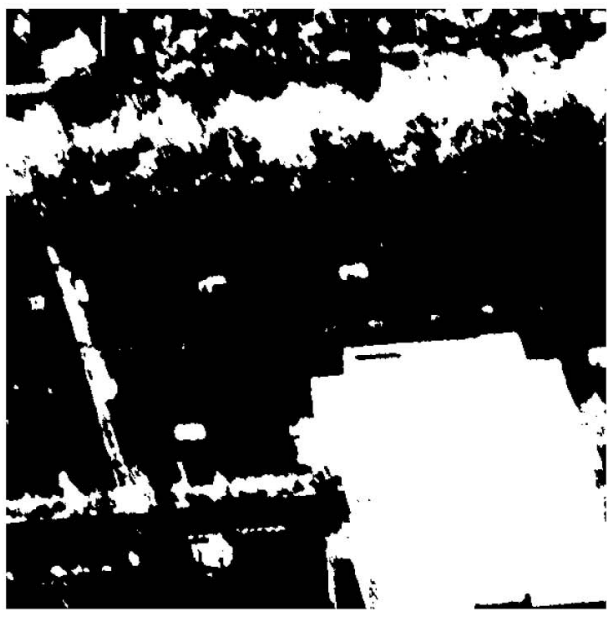

(d)

Fig. 9. Shadow detection on DLR 3K Camera data (shadows from medium-rise buildings, residential urban area). (a) RGB band composition. (b) Tsai method [2]. (c) Shorter method [24]. (d) Proposed method. Shadow masks are represented in "white."

TABLE IV

Shadow Detection Accuracy Numerical Assessment (Best Scores Are Marked in Bold)

\begin{tabular}{|c||c|c||c|c||c|c|}
\hline \multicolumn{1}{|c||}{} & \multicolumn{2}{c||}{ Tsai method [2] } & \multicolumn{2}{c||}{ Shorter method [24] } & \multicolumn{2}{c|}{ Proposed method } \\
\hline Sensor & $D R$ & $F A R$ & $D R$ & $F A R$ & $D R$ & $F A R$ \\
\hline Landsat 7 ETM+ (Figure 4) & 0.80 & 0.19 & $\mathbf{0 . 9 9}$ & 0.21 & $\mathbf{0 . 9 9}$ & $\mathbf{0 . 0 0}$ \\
\hline IKONOS (Figure 5) & 0.53 & 0.51 & 0.96 & 0.12 & $\mathbf{0 . 9 9}$ & $\mathbf{0 . 0 1}$ \\
\hline IKONOS (Figure 6) & 0.36 & 0.03 & 0.2 & $\mathbf{0 . 0 0}$ & $\mathbf{0 . 9}$ & 0.05 \\
\hline WorldView-2 (Figure 7) & 0.57 & 0.36 & $\mathbf{0 . 9 9}$ & 0.48 & 0.97 & $\mathbf{0 . 0 1}$ \\
\hline DLR 3K Camera (Figure 8) & 0.66 & 0.43 & 0.82 & 0.17 & $\mathbf{0 . 9 7}$ & $\mathbf{0 . 1 2}$ \\
\hline DLR 3K Camera (Figure 9) & 0.70 & 0.27 & 0.96 & 0.19 & $\mathbf{0 . 9 8}$ & $\mathbf{0 . 0 1}$ \\
\hline Overall accuracy & 0.60 & 0.3 & 0.82 & 0.2 & $\mathbf{0 . 9 7}$ & $\mathbf{0 . 0 3}$ \\
\hline
\end{tabular}

better detection of shadows but together with the very high FAR value $(F A R=0.48)$; therefore, the presented method is preferable. Another problem of shadow detection in very high resolution data is moving objects. A high FAR score is partly caused by a wrong assignment of moving objects as shadow.

Many factors influencing image acquisition parameters also influence the accuracy of shadow detection. The main factors are as follows.

1) Daily and seasonal changes of solar illumination. The change of the illumination spectra leads to a change of the reflected spectra and, therefore, to a change of the chromaticity of the objects. The detection of shadows according to the acquired spectral or color properties of the scene and comparison with prior expectations may provide errors in the detection.

2) Geographic position, atmospheric composition (aerosol optical thickness, water vapor column, etc.), and insolation. Change of geographic position, as well as elevation, leads to changes in direct-sunlight spectral distribution. The Sun illumination spectra are influenced by the atmospheric composition. Moreover, the sunlight passing through the atmosphere is attenuated. 
3) Content of the scene. For example, highly reflecting objects in a shadowed area can be interpreted as nonshadow by the detection methods based on invariant color spaces.

Therefore, many of the methods with straightforward employment of invariant color spaces may fail because of high variability of the image intensity in shadowed and illuminated regions. The properties of the illumination sources, as well as the properties of the illumination in shadow area, together with the adaptive calculation of the method's parameter set, can only allow robust and stable detection of shadows.

In comparison to the tricolor attenuation model [10], our method adaptively calculates all the parameter set values from the input image and does not have restrictions on the lighting conditions, i.e., there is no need to recalculate parameters or extend to other lighting environments.

This method is easy for deployment as a procedure for a system on remotely sensed image processing and interpretation. The complexity of the method is dependent primarily on the algorithm for shadow border search and on the method for the temperature calculation. The selection of the proper method for shadow border search, as well as automatic selection of the initial parameters for the Brent's method, allows one to run this method with very low execution time.

Further application of this method has been performed for reduction of errors in city digital elevation model update (wrong boundaries of buildings can be detected due to the building cast shadow and self-shadow), change detection (shadows from clouds and buildings), and for classification of optical data (specific classes among which a shadow class is outlined).

\section{CONCLUSION}

An alternative method for shadow detection for remotely sensed optical data has been developed. This method is based on the physical properties of illumination source and employs the blackbody radiator model for the description of the illumination process. Robustness and high accuracy of shadow detection are reached by the adaptive nature of the method. The adaptiveness of the method consists in the calculation of the parameter set values for a particular input image. This allows one to extract all types of shadows from a single image.

High scores of statistical assessment rates were obtained on different remotely sensed imageries influenced by the variations of illumination, acquisition time, Lat/Lon coordinates, the Sun elevation, atmospheric conditions, etc. High DRs were obtained on the processed data sets of multispectral visible and nearinfrared images.

It should be noted that the application of the method is not limited to remotely sensed data. The method can easily be applied to other imagery from different sources and in the areas of image recognition (object tracking, robot navigation, etc.).

\section{ACKNOWLEDGMENT}

We would like to thank European Space Imaging (EUSI) for the collection and provision of Digitalglobe WorldView-2 and IKONOS data over Munich city.

\section{REFERENCES}

[1] D. C. Knill, P. Mamassian, and D. Kersten, "Geometry of shadows," J. Opt. Soc. Amer. A, Opt. Image Sci., vol. 14, no. 12, pp. 3216-3232, Dec. 1997.

[2] V. J. D. Tsai, "A comparative study on shadow compensation of color aerial images in invariant color models," IEEE Trans. Geosci. Remote Sens., vol. 44, no. 6, pp. 1661-1671, Jun. 2006.

[3] K. L. Chung, Y. R. Lin, and Y. H. Huang, "Efficient shadow detection of color aerial images based on successive thresholding scheme," IEEE Trans. Geosci. Remote Sens., vol. 47, no. 2, pp. 671-682, Feb. 2009.

[4] E. Salvador, A. Cavallaro, and T. Ebrahimi, "Cast shadow segmentation using invariant color features," Comput. Vis. Image Understand., vol. 95, no. 2, pp. 238-259, Aug. 2004.

[5] Y. Wang and S. Wang, "Shadow detection of urban aerial images based on partial differential equations," in Proc. ISPRS Congr., Comm. II, Jul. 3-11, 2008, vol. XXXVII, pp. 325-328, Part B2.

[6] J. Yao and Z. M. Zhang, "Hierarchical shadow detection for color aerial images," Comput. Vis. Image Understand., vol. 102, no. 1, pp. 60-69, Apr. 2006.

[7] M. F. Tappen, W. T. Freeman, and E. H. Adelson, "Recovering intrinsic images from a single image," IEEE Trans. Pattern Anal. Mach. Intell., vol. 27 , no. 9, pp. 1459-1472, Sep. 2005.

[8] H. Jiang and M. S. Drew, "Tracking objects with shadows," in Proc. SPIE_Image and Video Communications and Processing 2003, May 7, 2003, pp. 512-521.

[9] T.-P. Wu and C.-K. Tang, "A Bayesian approach for shadow extraction from a single image," in Proc. IEEE Int. Conf. Comput. Vis., Oct. 17-21, 2005, vol. 1, pp. 480-487.

[10] J. Tian, J. Sun, and Y. Tang, "Tricolor attenuation model for shadow detection," IEEE Trans. Image Process., vol. 18, no. 10, pp. 2355-2363, Oct. 2009.

[11] A. M. Polidorio, F. C. Flores, N. N. Imai, A. M. G. Tommaselli, and C. Franco, "Automatic shadow segmentation in aerial color images," in Proc. Brazilian Symp. Comput. Graph. Image Process., Los Alamitos, CA, Oct. 12-15, 2003, pp. 270-277.

[12] S. Chakraborti, "Verification of the Rayleigh scattering cross section," Amer. J. Phys., vol. 75, no. 9, pp. 824-826, Sep. 2007.

[13] J. A. Marchant and C. M. Onyango, "Spectral invariance under daylight illumination changes," J. Opt. Soc. Amer. A, Opt. Image Sci., vol. 19, no. 5, pp. 840-848, May 2002.

[14] R. Kawakami, J. Takamatsu, and K. Ikeuchi, "Color constancy from blackbody illumination," J. Opt. Soc. Amer. A, Opt. Image Sci., vol. 24, no. 7, pp. 1886-1893, Jul. 2007.

[15] G. D. Finlayson and S. D. Hordley, "Color constancy at a pixel," J. Opt. Soc. Amer. A, Opt. Image Sci., vol. 18, no. 2, pp. 253-264, Feb. 2001.

[16] N. Otsu, "A threshold selection method from gray level histograms," IEEE Trans. Syst., Man, Cybern., vol. SMC-9, no. 1, pp. 62-66, Jan. 1979.

[17] W. H. Press, S. A. Teukolsky, W. T. Vetterling, and B. P. Flannery, Numerical Recipes in $C$ : The Art of Scientific Computing. New York: Cambridge Univ. Press, 1992.

[18] I. Priest, "The colorimetry and photometry of daylight and incandescent illuminants by the method of rotatory dispersion," J. Opt. Soc. Amer, vol. 7, no. 12, pp. 1175-1209, Dec. 1923.

[19] G. D. Finlayson, S. D. Hordley, and M. S. Drew, "Removing shadows from images," in Proc. 7th Eur. Conf. Comput. Vis., May 28-31, 2002, vol. 2353, pp. 823-836.

[20] G. D. Finlayson, S. D. Hordley, and M. S. Drew, "Removing shadows from images using Retinex," in Proc. IS\&T/SID 10th Color Image Conf.: Color Sci. Eng. Syst. Technol. (Soc. Inf. Display), Scottsdale, AZ, Nov. 2002, pp. 73-79.

[21] T. Gevers and H. Stokman, "Classifying color edges in video into shadowgeometry, highlight, or material transitions," IEEE Trans. Multimedia, vol. 5, no. 2, pp. 237-243, Jun. 2003.

[22] F. Kurz, D. Rosenbaum, U. Thomas, J. Leitloff, G. Palubinskas, K. Zeller, and P. Reinartz, "Near real time airborne monitoring system for disaster and traffic applications," in Proc. ISPRS Workshop, Hannover, Germany, Jun. 2-5, 2009.

[23] A. Prati, I. Mikic, M. Trivedi, and R. Cucchiara, "Detecting moving shadows: Algorithms and evaluation," IEEE Trans. Pattern Anal. Mach. Intell., vol. 25, no. 7, pp. 918-923, Jul. 2003.

[24] N. Shorter and T. Kasparis, "Automatic vegetation identification and building detection from a single nadir aerial image," Remote Sens., vol. 1, no. 4 , pp. 731-757, Oct. 2009. 


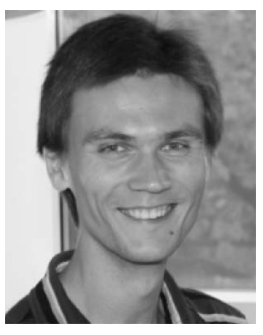

Aliaksei Makarau received the Diploma (Dipl.Ing.) degree in computer science from the Belarusian State University of Informatics and Radioelectronics, Minsk, Belarus, in 2003 and the Ph.D. degree in technical sciences from the United Institute of Informatics Problems, Minsk, in 2008. His dissertation was on fast methods for multispectral image fusion and processing.

He is currently a Postdoctoral Fellow with the Department of "Photogrammetry and Image Analysis," Remote Sensing Technology Institute (IMF), German Aerospace Center (DLR), Wessling, Germany. His research interests include multimodal data fusion, pattern recognition, and automatic classification.

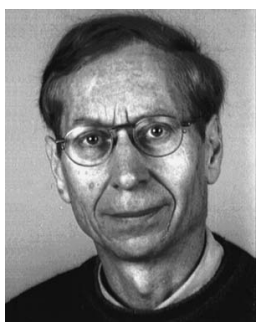

Rudolf Richter received the M.Sc. degree in physics from the Technical University of Munich, Munich, Germany, in 1973 and the Ph.D. (Dr. Ing.) degree in engineering from the Technical University of Dresden, Dresden, Germany, in 1991.

$\mathrm{He}$ is currently a Senior Scientist with the German Aerospace Center (DLR), Wessling, Germany, conducting concept development, modeling, and simulation of airborne/spaceborne hyperspectral instruments. It involves advanced techniques and interaction with scientists associated with theory and remote sensing experiments. He developed the ATCOR model, one of the standard codes for atmospheric and topographic corrections of multi/hyperspectral imagery used at universities and research laboratories. His current work focuses on the design of fully automatic processing chains for the evaluation of remotely sensed optical data from the visible to the thermal spectral region.

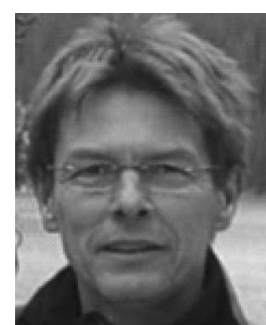

Rupert Müller received the Dipl.-Phys. degree from the Ludwig Maximilians University of Munich, Munich, Germany, in 1985

$\mathrm{He}$ is currently a Team Leader of the "Processors and Traffic Monitoring" Group, Remote Sensing Technology Institute, German Aerospace Center (DLR), Wessling, Germany, and is responsible for the Environmental Mapping and Analysis Program processing, calibration, and validation part within the ground segment, as well as several European Space Agency projects like "Prototype Processor for ALOS Optical Data." His main research interests include photogrammetric evaluation of spaceborne satellite data and digital image processing.

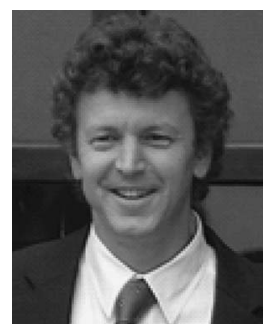

Peter Reinartz received the Diploma (Dipl.-Phys.) degree in theoretical physics from the University of Munich, Munich, Germany, in 1983 and the Ph.D. (Dr--Ing) degree in civil engineering from the University of Hannover, Hannover, Germany, in 1989. His dissertation was on the statistical optimization of classification methods for multispectral image data.

$\mathrm{He}$ is currently the Head of the Department of "Photogrammetry and Image Analysis," Remote Sensing Technology Institute (IMF), German Aerospace Center (DLR), Wessling, Germany, and holds a professorship on geoinformatics at the University of Osnabrueck, Osnabrueck, Germany. He has more then 20 years of experience in image processing and remote sensing and over 150 publications in these fields. He is also engaged in using remote sensing data for disaster management and using high-frequency time series of airborne image data for real time application in the case of disasters, as well as for traffic monitoring. His main interests are in direct georeferencing, stereo photogrammetry and data fusion, generation of digital elevation models, and interpretation of VHR image data from sensors like WorldView, GeoEye a.o. 